\title{
sciendo
}

\section{Is Test Standardization Important when Arm and Leg Muscle Mechanical Properties are Assessed Through the Force-Velocity Relationship?}

\author{
by \\ Marko Cosic ${ }^{1}$, Sasa Djuric ${ }^{1}$, Milena Z. Zivkovic ${ }^{1}$, Aleksandar Nedeljkovic ${ }^{1}$, \\ Bojan Leontijevic ${ }^{1}$, Slobodan Jaric ${ }^{1,2,3}$
}

The force-velocity (F-V) relationship observed in multi-joint tasks proved to be strong and approximately linear. Recent studies showed that mechanical properties of muscles: force $(F)$, velocity $(V)$ and power $(P)$ could be assessed through the F-V relationship although the testing methods have not been standardized. The aim of the present study was to evaluate and compare F-V relationships assessed from two tests performed on a modified Smith machine that standardizes kinematics of the movement pattern. Fifteen participants were tested on the maximum performance bench press throws and squat jumps performed against a variety of different loads. In addition, their strength properties were assessed through maximum isometric force (Fiso) and one repetition maximum (1 RM). The observed individual F-V relationships were exceptionally strong and approximately linear ( $r=0.98$ for bench press throws; $r=0.99$ for squat jumps). F-V relationship parameter depicting maximum force (F0) revealed high correlations with both Fiso and 1 $R M$ indicating high concurrent validity $(p<0.01)$. However, the generalizability of $F-V$ relationship parameters depicting maximum force (F0), velocity (V0) and power (PO) of the tested muscle groups was inconsistent and on average low (i.e. FO; $r=-0.24$ ) to moderate (i.e. $V 0$ and P0; $r=0.54$ and $r=0.64$, respectively; both $p<0.05$ ). We concluded that the F-V relationship could be used for the assessment of arm and leg muscle mechanical properties when standard tests are applied, since the typical outcome is an exceptionally strong and linear F-V relationship, as well as high concurrent validity of its parameters. However, muscle mechanical properties could be only partially generalized across different tests and muscles.

Key words: generalizability, concurrent validity, parameter, power, load.

\section{Introduction}

To be successful in various sports, the athletes should be able to quickly accelerate either their bodies or parts of the body from the resting position (e.g., sprints, jumps, throws, shots, etc.). This ability in ballistic movements is directly related to mechanical properties of muscles, and notably to power capabilities. Since power is the product of force and velocity, muscle mechanical properties also include force and velocity capabilities which represent the ability to develop the high level of force and the ability to contract the muscles at high velocity (Morin and Samozino, 2018). The assessment of muscle mechanical properties, specifically the $\mathrm{F}, \mathrm{V}$, and $\mathrm{P}$ output, could be a good indicator for coaches to improve athletes' performance in intended direction. Since the standard testing procedures typically involve the application of only one external load, the informational value of the assessed outcomes is limited. Namely, the

1 - University of Belgrade, Faculty of Sport and Physical Education, The Research Center, Belgrade, Serbia.

2 - University of Delaware, Department of Kinesiology and Applied Physiology E Biomechanics and Movement Science Graduate Program, Rust Arena, Newark 19716, DE, USA.

3 - Department of Human Motor Behavior, The Jerzy Kukuczka Academy of Physical Education in Katowice, Katowice, Poland. 
different muscle mechanical properties cannot be discerned from a single outcome of the testing procedures (Jaric, 2015). To obtain more informational assessment of muscle mechanical properties, the standard testing procedures should be extended by including different tests, which could potentially lead to both physical and mental fatigue (Carriker, 2017). As a consequence, the reliability of the standard tests could be significantly decreased due to such improper testing conditions, thus questioning the validity of the standard tests as well (McMaster et al., 2014).

Recent studies suggest that the approximately linear and strong force-velocity (FV) relationship obtained in multi-joint functional movements could be one of the solutions of the discussed problem (García-Ramos et al., 2016; Jaric, 2015; Nikolaidis, 2012; Sánchez-Medina et al., 2014). Namely, experimental findings indicate that the F-V relationship could be used for the assessment of muscle mechanical properties in various multi-joint functional movement tasks such as the bench press (Cronin et al., 2003; Sreckovic et al., 2015; Golas et al., 2017), bench pull (Zivkovic et al., 2017b), throws (Van Den Tillaar and Ettema, 2004), vertical jumps (Cuk et al., 2014; Jiménez-Reyes et al., 2017; JiménezReyes et al., 2014; Samozino et al., 2014), cycling (Jaskolska et al., 1999; Zivkovic et al., 2017b), or running (Dobrijevic et al., 2017; Morin et al., 2010; Rabita et al., 2015). Moreover, the F-V relationship has been also used to assess the outcomes of conducted training programs (Djuric et al., 2016). However, some studies revealed weaker and, therefore, not necessarily linear F-V relationships of multi-joint functional movements (Allison et al., 2013; Feeney et al., 2016; Hahn et al., 2014; Limonta and Sacchi, 2010). This discrepancy might be at least partly attributed to different applied methodologies, which require further effort on their standardization.

Anyway, when the F-V relationship is used for the assessment of muscle mechanical properties more external loads should be added during the testing procedure to provide a range of $\mathrm{F}$ and $\mathrm{V}$ data that would allow for applying a multiple-load model (Zivkovic et al., 2017b). The model is based on regression modelling that inevitably reveals parameters of maximum $\mathrm{F}$ (i.e., the F-intercept; $\mathrm{F}_{0}$ ), maximum $\mathrm{V}$ (i.e., the $\mathrm{V}$ intercept; $\left.\mathrm{V}_{0}\right)$, and maximum $\mathrm{P}\left(\mathrm{P}_{0}=\mathrm{F}_{0} \cdot \mathrm{V}_{0} / 4\right)$.
The obtained F-V relationship parameters represent the external $\mathrm{F}, \mathrm{V}$, and $\mathrm{P}$ output of the tested muscles. Of particular importance is the fact that F-V relationship parameters proved to be highly reliable and at least moderately valid, particularly when their concurrent validity was assessed through correlation with correspondent variables directly measured using standard tests (Jaric, 2015). Nevertheless, although several studies have investigated concurrent validity of the F-V relationship parameters, the results proved to be rather inconsistent. For example, the concurrent validity of $\mathrm{F}_{0}$ was moderate-to-high (Cuk et al., 2014; Driss et al., 2002; Vandewalle et al., 1987), while in some other studies it was low or even non-significant (Rahmani et al., 2001; Ravier et al., 2004; Yamauchi and Ishii, 2007) regarding the directly measured muscle strength. In addition to the diversity of applied methodologies, the other possible reasons for different findings could originate either from inherent differences among various tests or the involved muscles.

The standardization of the testing procedures is a prerequisite for the internal validation of experimental findings. It might be presumed that using the Smith machine could standardize testing conditions when various muscle mechanical properties need to be assessed. First, the Smith machine is a testing device that allows for safe performance of dynamic exercise while recording the relevant kinematic data (Wilson et al., 1996). Second, it allows to conduct testing under more controllable conditions that presumably reduce kinematic variability by constraining the tested movement to the vertical direction. As a consequence, the reliability of testing performed on a Smith machine could be relatively high. Third, safer and more controlled conditions enable applying wider range of the external load when jumping or throwing is the tested movement pattern. Namely, recent studies that investigated F-V relationships in jumping often used loaded vests on force plates (Markovic et al., 2013; Pazin et al., 2013; Zivkovic et al., $2017 \mathrm{~b}$ ), where the range of magnitude of applied loads was limited and adaptation of the movement pattern could occur (Mandic et al., 2015; Markovic et al., 2014). Therefore, it is not surprising that the Smith machine has been often used in standard tests which include lifting, 
pulling, throwing and jumping (Djuric et al., 2016; García-Ramos et al., 2016; Sreckovic et al., 2015; Zivkovic et al., 2017a).

If the testing conditions are standardized, such is when a Smith machine is used, one might ask whether the mechanical properties recorded from one test could be generalized across other muscles (e.g., arm or leg muscles). The relationships between the muscle mechanical properties assessed through $\mathrm{F}_{0}, \mathrm{~V}_{0}$ and $\mathrm{P}_{0}$, obtained from different tests have been assessed in only one study (Zivkovic et al., 2017a). The results showed that the muscle mechanical properties observed through F-V relationship parameters obtained from one test could be only partially generalized to other tests and muscles. However, functional tests for upper and lower body parts were conducted under different testing conditions (e.g., different contraction regimes). Therefore, the study designed to investigate the generalizability of the $\mathrm{F}-\mathrm{V}$ relationship parameters (i.e., $\mathrm{F}_{0}, \mathrm{~V}_{0}$ and $\mathrm{P}_{0}$ ) obtained from different muscles tested in similar standardized testing conditions could provide a novel insight into this unresolved issue filling the observed gap in the literature.

To address the discussed problem, we designed a study to evaluate and compare F-V relationships assessed from two standard tests performed on a modified Smith machine: bench press throws and squat jumps. Our first hypothesis was that the F-V relationships explored on different muscle groups (i.e., arm vs. leg muscle groups) would be strong and approximately linear. The second hypothesis was that the concurrent validity of parameters $F_{0}$ observed from the linear F-V relationships with directly measured muscle strength would be high in both standard tests. The third hypothesis was that the muscle mechanical properties obtained through the F-V relationship parameters would be generalizable across two applied standard tests. The findings were expected to contribute to our understanding of both the mechanical properties and function of human muscular system, as well as to enable further development of the methods for routine testing of muscle strength, velocity, and power properties. In addition, the standardization of testing conditions could provide the novel insight into two potentially unresolved issues regarding the assessment of muscle mechanical properties through the $\mathrm{F}-\mathrm{V}$ relationship: (1) the concurrent validity of $F_{0}$ that showed to be rather inconsistent due to either diverse applied methodologies, or the involved muscles, or inherent differences among various tests; and (2) the generalizability of F-V parameters that showed to be only partial when functional tests for upper and lower body parts were conducted under different contraction regimes.

\section{Methods}

\section{Participants}

We recruited 15 male physical education students (age $20.9 \pm 2.0$; body mass $82.5 \pm 5.9 \mathrm{~kg}$, body height $185.2 \pm 4.9 \mathrm{~cm}$, percent body fat $10.1 \pm$ $4.2 \%$, BMI $24.0 \pm 1.6 \mathrm{~kg} / \mathrm{m}^{2}$ ). To simplify the loading procedure, we selected (out of the larger pool of 40 participants) only those who had an extensive experience in resistance training and those with one repetition maximum (1 RM) bench press between 90 and $110 \mathrm{~kg}(98.3 \pm 6.3 \mathrm{~kg})$ and with $1 \mathrm{RM}$ squat between 130 and $150 \mathrm{~kg}(138.3 \pm$ $8.2 \mathrm{~kg}$ ). The participants were physically active through their standard academic curriculum that included at least 6 (no more than 8) activity classes per week that involved both low and high intensity exercises. They reported no chronic diseases, heart problems, or recent injuries to their musculoskeletal apparatus. During the study period none of participants was an active athlete and they were instructed to avoid any strenuous exercise over the course of the study. The participants were informed regarding the potential risks associated with the applied testing protocol and gave written informed consent to the experiments. Both the consent and the experimental procedure were in accordance with the Declaration of Helsinki and approved by the Institutional Review Board of the University of Belgrade - Faculty of Sport and Physical Education.

\section{Experimental protocol}

The testing protocol consisted of the assesment of both the maximal isometric force (Fiso) and $1 \mathrm{RM}$ for the bench press and squat, as well as of the assessment of the kinematical parameters in the bench press throw and the squat jump. Both tests were conducted on the same modified Smith mashine (Figure 1). Each participant completed 4 testing sessions separated 
by 3 or more days of rest. The first testing session included anthropometric measurements, initial familiarization with the motor tasks, and the assessment of the $F_{\text {iso }}$ bench press and the $F_{\text {iso }}$ squat. The participants' body mass and body height were assessed with a digital scale and a standard anthropometer, respectively, and their percent body fat was assessed using a bioelectric impedance method (In Body 720; InBody, Cerritos, CA, USA). The second testing session included final familiarization with the motor tasks and the assessment of the $1 \mathrm{RM}$ bench press and the squat. The third and fourth sessions cosisted of testing either bench press throws or squat jumps where their sequence was randomized across the participants. Testing included 2 trials under each of the 7 external loads ranging from 20 to $80 \mathrm{~kg}$. All testing sessions were preceded by a standard warm up procedure - a 5 min ride on a bicycle ergometer, $8 \mathrm{~min}$ of mobilization exercises followed by the specific warm up depending on the test (see further text for details).

$F_{\text {iso }}$ testing

Fiso testing used a modified Smith machine that was provided with one additional upper bar. This additional upper bar could be fixed on a selected height and was attached to the barbell over a calibrated strain-gauge force transducer (Hottinger, Type S9, range $10 \mathrm{kN}$; linearity better than $1 \%$, tensile/compressive force sensitivity $2 \mathrm{mV} / \mathrm{N})$.

For the assessment of the Fiso bench press the barbell was positioned $1 \mathrm{~cm}$ above the participant's chest. The participants were instructed to perform the maximum isometric bench press while maintaining shoulders at $90^{\circ}$ of abduction to ensure a stable pattern of the shoulder and elbow joints throughout testing (Newton et al., 1997). No bouncing or arching of the back was allowed. The feet and pelvis had to remain in contact with the bench.

For the assessment of the $F_{\text {iso }}$ squat the barbell was positioned on shoulders that enabled the $90^{\circ}$ angle in knees of each participant, while feet were shoulder width apart. The projection of the barbell went through participant thighs, lower legs and feet (Figure 1b). No arching of the back was allowed. For measuring $F_{\text {iso }}$ in both tests participants performed $4 \mathrm{~s}$ of maximum voluntary contraction during pushing the barbell attached to the fixed additional upper bar. Three trials were performed with the rest period of $3 \mathrm{~min}$ in between. The specific warm up for each testing task included a progressive increase of force production.

\section{RM testing}

The starting position of the barbell for 1 $\mathrm{RM}$ in the bench press and squat was the same as

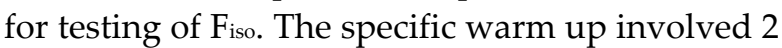
x 6 repetitions of the bench press /squat at $40 \%$ and $60 \%$ of the estimated $1 \mathrm{RM}$ (Fiso testing was used as a landmark). Two to three trials were performed for each task until the participant was unable to reach the full extension of the arms (bench press) or legs (squat) with the highest possible load. The previous trial was taken as 1 RM. The rest interval between the trials was 4 $\min$.

\section{Bench press throw and squat jump testing}

The starting position of the barbell for the bench press throw and squat jump was the same

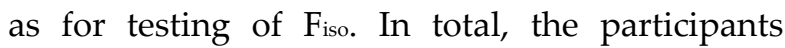
completed 14 trials of both the bench press throw and the squat jump ( 2 trials $\times 7$ loads) that were randomized regarding the load magnitude. The minimum load of $20 \mathrm{~kg}$ consisted of the barbell, while the weight plates were added for higher loads. The applied external loads in both tests (i.e., bench press throw and squat jump) were 20, 30, $40,50,60,70$, and $80 \mathrm{~kg}$. For bench press throws the total mass lifted included $5 \%$ of participant's body mass, the barbell and weight plates, while squat jumps involved $88 \%$ of participant's body mass, the barbell and weight plates (calculated by the Dempster's model; Dempster \& United States. Wright Air Development Division). To avoid fatigue, the rest period between 2 consecutive trials was $1 \mathrm{~min}$, while the rest between the different loading conditions was about $4 \mathrm{~min}$. Regarding the tested task, the participants were instructed "to apply their full strength against the barbell and throw it/jump as high as possible". Although the mechanical stops prevented lowering the barbell under its initial position, 2 spotters caught the barbell during its descending trajectory. The specific warm up consisted of $2 \times 2$ throws/jumps at $20 \mathrm{~kg}$ of the external load.

\section{Data processing and analyses}

The vertical displacement of the barbell was recorded with 3D infra-red cameras (Qualisys $\mathrm{AB}$, Gothenburg, Sweden) sampled at a rate of 240 $\mathrm{Hz}$ with a recursive Butterworth $10 \mathrm{~Hz}$ low-pass 
filter. The custom-made LabVIEW software was used to assess $\mathrm{F}$ and $\mathrm{V}$ from bench press throws and squat jumps. $V$ and acceleration of the barbell were calculated from the first and second derivative of the position, respectively, while $\mathrm{F}$ was calculated as a sum of the weight (i.e., mass times gravity acceleration) and inertia (mass times acceleration) of the total mass lifted (Djuric et al., 2016; Sreckovic et al., 2015; Zivkovic et al., 2017a; Zivkovic et al., 2017b). Note that F and V were calculated as average values during the concentric phase of the movement - from the time the barbell started moving until acceleration of the barbell dropped to $-9.81 \mathrm{~m} / \mathrm{s}^{2}$ (Cronin et al., 2003; Djuric et al., 2016; Sreckovic et al., 2015).

\section{Statistical analyses}

The Kolmogorov-Smirnov test revealed that none of the dependent variables significantly deviated from normal distribution. Descriptive statistics were calculated as means, standard deviations, and the coefficient of variation. Both linear and polynomial $2^{\text {nd }}$ order regression models were used to assess the individual and averaged values across the participants' F-V relationships. The corresponding $95 \%$ confidence intervals (95\% $\mathrm{CI})$ were thereafter calculated. Since there were no significant differences between the 2 models (see Results for details), further analysis was based only on the outcomes of the linear model. Pearson's correlations and independent t-tests were employed to obtain the concurrent validity of $F_{0}$ with respect to the directly recorded strength (i.e. $F_{\text {iso }}$ and $1 \mathrm{RM}$ ) recorded in the bench press and the squat. The level of statistical significance was set at $p<0.05$. All statistical analyses were performed using SPSS 21 (IBM, Armonk, NY).

\section{Results}

Figure 2 shows the linear models of F-V relationships assessed in two different tests - the bench press throw and the squat jump. The individual data points represent $\mathrm{F}$ and $\mathrm{V}$ variable types for each individual load averaged across the participants. Regressions revealed exceptionally high correlation coefficients $(r=0.98$ for bench press throws, $r=0.99$ for squat jumps) suggesting that the observed F-V relationships were strong and linear. This finding was confirmed when the applied linear regressions were compared to the polynomial 2nd order ones. Namely, the polynomial regressions applied on the individual data points collected in bench press throws and squat jumps revealed correlation coefficients $(95 \%$ confidence intervals) of $\mathrm{r}=0.98(0.87-0.99)$ and $\mathrm{r}$ $=0.99(0.96-1.00)$, respectively. Note that the corresponding correlation coefficients obtained from linear regression models were within the $95 \%$ confidence intervals of the polynomial ones indicating no significant differences between the outcomes of the two models.

Descriptive statistics (shown as mean and standard deviation) for F-V relationship parameters and directly measured strength variables are presented in Table 1. As expected, the values of the relationship parameters were higher when observed in squat jumps than in bench press throws. One can notice that the most prominent difference was observed in $\mathrm{P}_{0}$ which mostly originates from the parameter $\mathrm{F}_{0}$, but not from $V_{0}$. Therefore, the steepness of the regression slope in squat jumps was twice as high as compared to bench press throws. Regarding the coefficients of variation, the highest value was observed for $\mathrm{V}_{0}$ obtained from squat jumps (18.3\%). Regarding the linearity, the median individual correlation coefficient for bench press throws was $r=0.98$ (95\% CI was $0.97-0.99)$, while for squat jumps it was $\mathrm{r}=0.94(95 \% \mathrm{CI}$ was $0.94-$ 0.97).

Figure 3 shows the concurrent validity of $F_{0}$ for bench press throws and squat jumps, obtained through linear F-V regressions in regard to the directly measured strength. Results revealed high correlations with both $F_{\text {iso }}$ and 1 RM. The relationships were significant for all variables and tests $(p<0.01)$. The independent $t-$ test revealed that there were significant differences between $\mathrm{F}_{0}$ and $\mathrm{F}_{\text {iso }}$ as well as between $\mathrm{F}_{0}$ and $1 \mathrm{RM}$ in both tests, but not between $\mathrm{F}_{0}$ and $\mathrm{F}_{\text {iso }}$ in squat jumps $(p=0.38)$.

Regarding the generalizability of relationships of the parameters obtained from two different tests, the correlation coefficients observed between them ranged from weak and statistically non-significant for $\mathrm{F}_{0}(\mathrm{r}=-0.24)$ to moderate and statistically significant for $\mathrm{V}_{0}$ and $\mathrm{P}_{0}(\mathrm{r}=0.54$ and $\mathrm{r}$ $=0.64$, respectively; both $p<0.05$ ). 


\section{Table 1}

Descriptive statistics for individual F-V relationship parameters and directly measured strength variables.

\begin{tabular}{lllllll} 
& \multicolumn{5}{c}{ Bench press throw } & \multicolumn{5}{c}{ Squat jump } \\
& Mean & SD & CV \% & Mean & SD & CV\% \\
\hline$F_{\text {iso }}(\mathrm{N})$ & 1005 & 63 & 6.3 & 2211 & 136 & 6.2 \\
$1 \mathrm{RM}(\mathrm{N})$ & 1084 & 70 & 6.5 & 2069 & 92 & 4.4 \\
$\mathrm{~F}_{0}(\mathrm{~N})$ & 912 & 35 & 3.8 & 2260 & 164 & 7.2 \\
$\mathrm{~V}_{0}(\mathrm{~m} / \mathrm{s})$ & 2.9 & 0.2 & 6.2 & 3.8 & 0.7 & 18.3 \\
$\mathrm{P}_{0}(\mathrm{~W})$ & 670 & 55 & 8.2 & 2120 & 378 & 17.8 \\
\hline
\end{tabular}

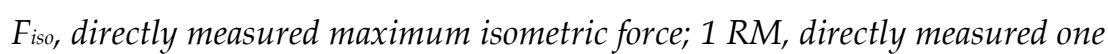
repetition maximum transformed from $\mathrm{kg}$ to $\mathrm{N}$; Fo, force intercept; $V_{0}$, velocity intercept, $\mathrm{P}_{0}$, maximum power; $\mathrm{CV} \%$, coefficient of variation.
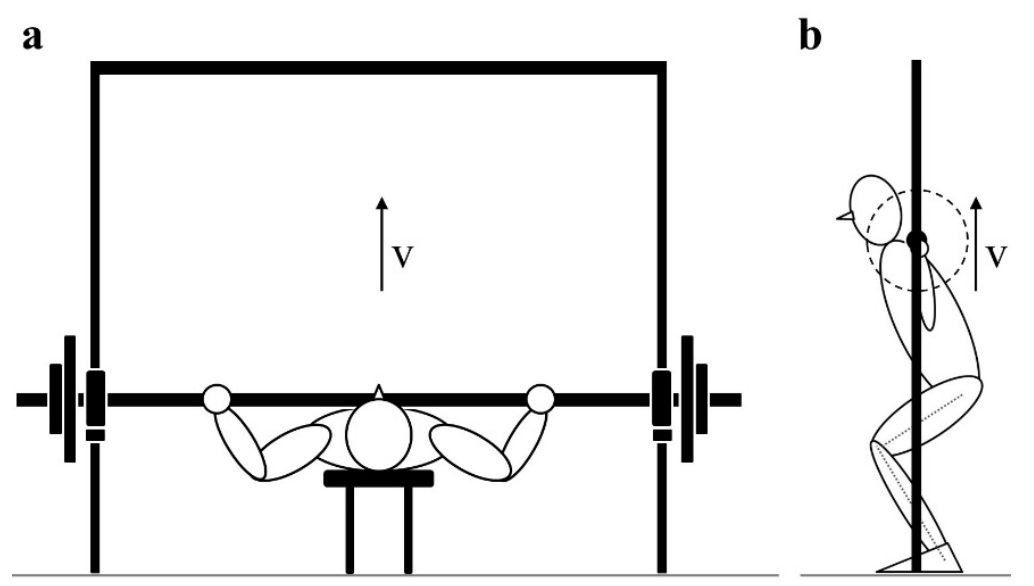

Figure 1

Illustration of the bench press throw (a) and the squat jump (b) performed on the same modified Smith machine. 


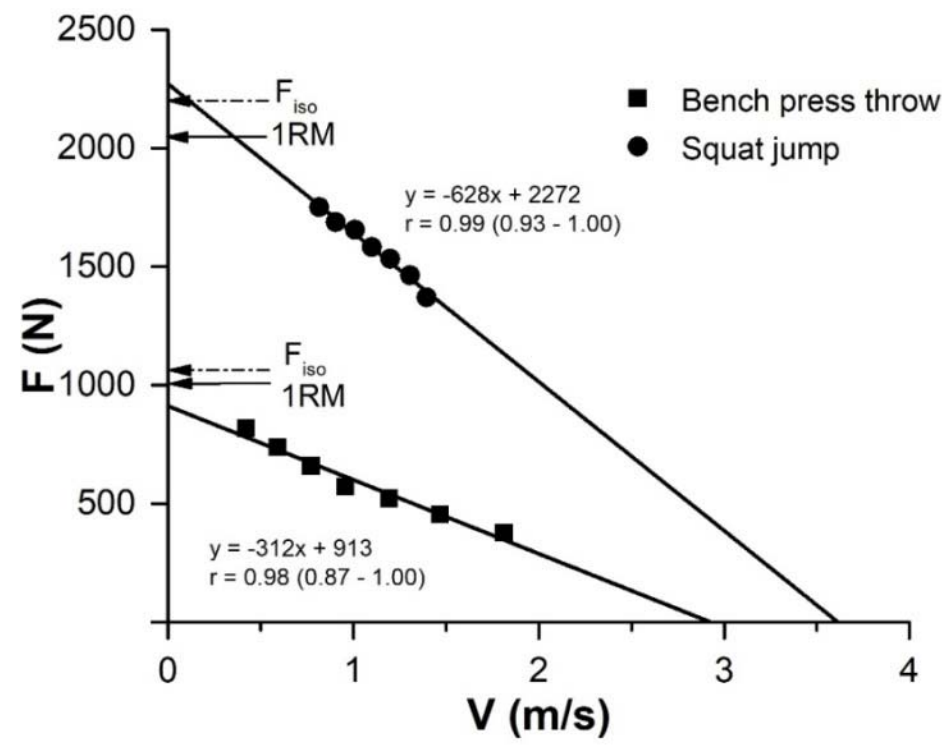

Figure 2

The linear regression models applied on the averaged across the participants' $F$ and $V$ variable types obtained from two different tests. Squares represent different magnitudes of the load for bench press throws, while circles represent the same for squat jumps. Fiso and 1 RM denote the recorded strength properties averaged across the participants. The regression equations are shown with the correlation coefficients and the corresponding 95\% CI.

\section{Figure 3}

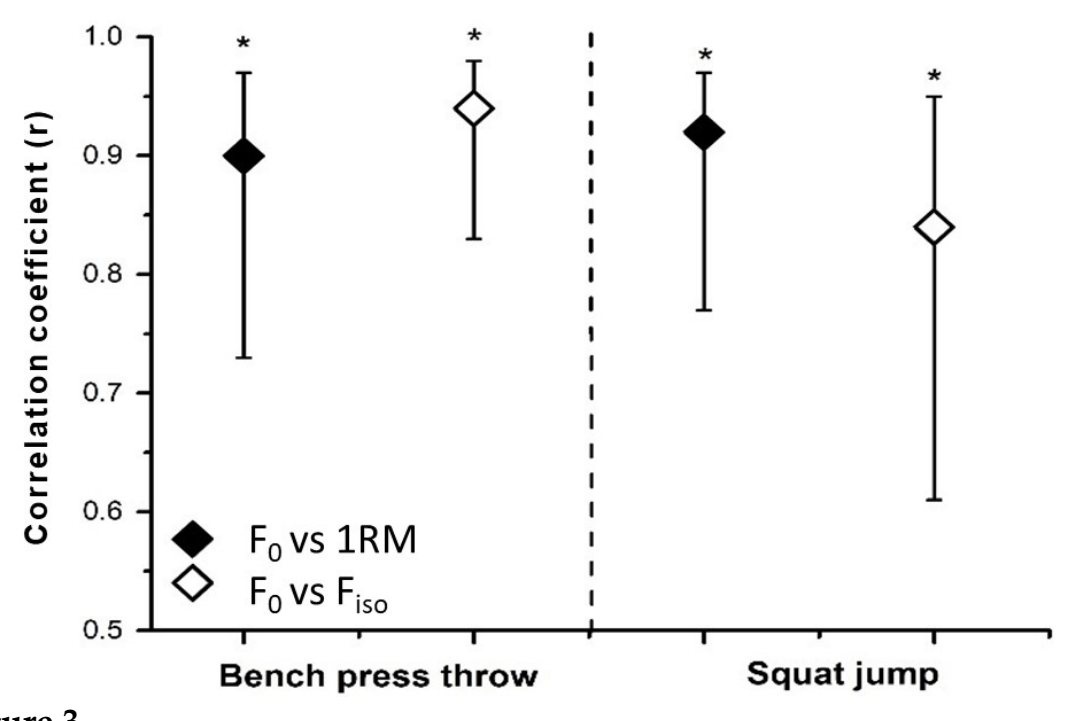

Concurrent validity of Fo obtained from linear F-V relationships assessed through

Pearson's correlations with the directly measured strength (i.e. Fiso and 1 RM).

Correlation coefficients (with the corresponding $95 \%$ CI) are shown for two tests and two variables $\left({ }^{*} p<0.01\right)$. 


\section{Discussion}

Within the present study, we explored and compared the F-V relationships of two standard multi-joint tests of muscle mechanical properties. Note that testing of muscle mechanical properties is more ecologically valid when the multi-joint movements are used since the majority of functional movements are performed in these conditions (e.g., running, jumping, throwing, kicking, etc.). In line with our first hypothesis, the findings suggest exceptionally strong and approximately linear F-V relationships in both the bench press throws and squat jumps. Regarding our second hypothesis, the results showed that $\mathrm{F}_{0}$ parameters observed from the linear F-V relationships in both tests, highly correlated with directly measured muscle strength (with both $\mathrm{F}_{\text {iso }}$ and $1 \mathrm{RM}$ ). Regarding our third hypothesis, we found that the muscle properties as assessed through the F-V relationship parameters obtained from muscles performing one test could be only partly generalized across other test and muscles.

Several potentially important novelties of the present study need to be addressed prior to discussing the obtained results. First, previous studies that compared F-V relationships obtained from different muscle groups have used different methodologies based on different types of loads (e.g., free weights, weighted wests and belts, elastic rubber bands) and different contraction regimes (e.g., eccentric-concentric vs. concentric only). The testing protocols performed on a Smith machine that standardize kinematics of the movement pattern are supposed to provide similar outcomes when the arm and leg muscle mechanical properties are assessed through F-V relationship parameters. Second, the use of 3D infra-red cameras for the measurement of barbell vertical displacement in the bench press throw could be recognized as a 'gold standard' compared to the linear force transducer. On the other hand, the 'gold standard' could be a force plate when the squat jump is tested, although the velocity and power of the system center-of-mass are indirectly estimated from the recorded vertical ground reaction force (for detailed review on the assessment methodologies see McMaster et al., 2014). Anyway, the design of the present study was based on the same measurement method in both the bench press throw and the squat jump (i.e., the measurement of barbell vertical displacement with 3D infra-red cameras) due to our intention to maximally standardize testing conditions. Third, the range of loads in the present study was markedly increased, particularly when arm muscles were tested (i.e., 20 to $80 \mathrm{~kg}$ ), thus potentially providing higher reliability of the regression modeling outcomes (Perez-Castilla et al., 2018). Forth, the concurrent validity of $\mathrm{F}_{0}$ obtained from bench press throws and squat jumps was assessed with regard to both isometric and $1 \mathrm{RM}$ tests that were performed from the same starting position as the referent one. Note that isometric and $1 \mathrm{RM}$ tests involve slightly different contraction regimes (i.e., isometric vs. slow concentric, respectively), thus the obtained results of concurrent validity might be generalized. Fifth, the possibility of generalization of mechanical properties of tested muscles to other muscle groups was investigated within the same standardized testing protocols using the same equipment (i.e., Smith machine). Finally, the experimental data were recorded from the same group of participants that included physically active individuals with a considerable previous experience in physical performance testing. In addition to the participants' experience, the standardized movement pattern could have minimized the effects of learning as well. This standardization was enabled due to the use of a Smith machine wherein the motion of the weighted barbell starts from a fixed position and it is vertically directed upward the sliding rails.

$\mathrm{F}-\mathrm{V}$ relationships explored within the present study proved to be strong and approximately linear. Moreover, the exceptionally high strength of the relationship was obtained from a wider range of loads, thus decreasing the probability of the alternative hypothesis that either a parabolic or other curvilinear models could be more appropriate. Note that our recent studies tested both linear and parabolic fit revealing no significant differences between those two regression models (Cuk et al., 2014; Sreckovic et al., 2015; Zivkovic et al., 2017a). The correlation coefficients obtained for the linear fit are comparable to the ones observed within the present study. Therefore, based on the presented set of data one might conclude that F-V relationships could be strong and approximately linear when observed in muscles performing bench press throws and squat jumps. 
Furthermore, one could speculate that the squat jump provides a stronger linear F-V relationship compared to the CMJ, since the starting standardized semi-squat position requires no adaptation of countermovement depth as a source of co-variation of the ankle, knee, hip, and other joint angles (Markovic et al., 2014). This could be of particular importance since both the joint angle co-variation and a lack of standardization of countermovement depth might confound the relationship between the recorded $\mathrm{F}$ and $\mathrm{V}$ (Mandic et al., 2015; Markovic et al., 2014). Namely, a moderate alteration of countermovement depth markedly affects both the $\mathrm{F}$ and $\mathrm{P}$ output of maximum, while the maximum $\mathrm{V}$ as assessed from the jump height remains virtually unchanged (Mandic et al., 2015). This finding supports our presumption that standard tests performed on a Smith machine could significantly improve testing conditions, when muscle mechanical properties are assessed through the F-V relationship. In contrast, the study of García-Ramos et al. (2017) recommends the CMJ over the squat jump, free weights over the Smith machine, and maximum over averaged variables due to both the stronger linearity of the F-V relationship and the higher reliability of the F-V parameters. Nevertheless, it is important to note that the different jumps tested within this study were performed on a force plate which could potentially confound the obtained results. Namely, it has been shown that the force plate underestimates the measured outcomes (PerezCastilla et al., 2017) compared to a linear transducer and possible 3D infra-red cameras used in our study. Apparently, further research on this important issue is needed.

To establish the concurrent validity of the Fo parameter of the studied F-V relationships, two already evaluated standard tests that directly assessed muscle strength (i.e., Fiso and $1 \mathrm{RM}$ ) have been selected (Abernethy et al., 1995; Wilson and Murphy, 1996). The face validity of the selected tests has been generally accepted (Abernethy et al., 1995). Higher concurrent validity of $F_{0}$ was expected since the external loads were altered through the wider range (i.e., 20 to $80 \mathrm{~kg}$ ) which could provide the relationship intercepts (i.e., $\mathrm{F}_{0}$ and $\mathrm{V}_{0}$ ) without using too distant extrapolations. The experimental findings supported this assumption indicating exceptionally strong correlation between the observed F-V relationship parameter (i.e., $\mathrm{F}_{0}$ ) and the outcomes of selected standard strength tests (i.e., $F_{\text {iso }}$ and 1 RM). Compared to the previous studies that investigated concurrent validity of $\mathrm{F}-\mathrm{V}$ parameters (Cuk et al., 2014; Driss et al., 2002; Ravier et al., 2004; Yamauchi and Ishii, 2007), the present study revealed on average higher correlation coefficients and a plausible assumption would be that the discussed finding could be attributed to the applied test standardization.

The frequent question to be asked here is whether the individual muscle properties to produce high F, V and $\mathrm{P}$ output could be generalized across different muscle groups. Therefore, we explored the relationship between the mechanical properties of arm and leg muscles assessed by 2 standard tests. The obtained results proved to be rather inconsistent. Specifically, the moderate relationship was observed between $\mathrm{V}_{0}$ as well as $\mathrm{P}_{0}$ obtained from 2 tests, while the relationship between their $\mathrm{F}_{0}$ was weak. These results are generally in line with our previous findings (Zivkovic et al., 2017a). The only exception could be regarding the relationship between $\mathrm{V}_{0}$ that proved to be somewhat stronger within the present study. The finding could be explained by the increased range of loads that provided more direct assessment of $\mathrm{V}_{0}$, rather than by an outcome of distinct extrapolation. Nevertheless, note that the observed moderate generalizability of the F-V parameters could be in line with recent studies revealing weak generalizability of strength measures across individual muscles of the muscular system (Bohannon, 2008) or functional movements (Pojednic et al., 2012). Note that different variables observed by employing different methods might strongly correlate within the same muscle, but weakly across the muscular system in general (Bozic et al., 2013; Prebeg et al., 2013). Moreover, the possible limitation of this study regarding the generalizability was the nature of participants training history. Namely, our participants were physical education students who were not only engaged in different sport activities on almost a daily basis, but also had some experience related to the conducted tests. Hence, future research should involve untrained participants. 


\section{Conclusions}

The standardization of testing conditions applied within the present study represents a novel approach that could provide more valid data when mechanical properties of arm and leg muscles have to be assessed. The obtained results revealed strong and linear F-V relationships in both standard multi-joint tests, while the concurrent validity of parameters $\mathrm{F}_{0}$ was high compared to directly measured muscle strength. These findings suggest that the F-V relationship could be used for the assessment of arm and leg muscle mechanical properties when standard tests are applied. Therefore, we may conclude that testing conditions based on the standardized kinematics of the movement pattern provided exceptional clear findings regarding both the linearity of the F-V relationship and concurrent validity of its parameters. However, muscle mechanical properties could be only partially generalized across different tests and muscles. This suggests that test standardization could be an important, but not the limited factor when arm and leg muscles are assessed through the F-V relationship.

\section{Acknowledgment}

The present study was conducted under the supervision of prof. Slobodan Jaric, who passed away during the review process of this paper. We wish to thank prof. Slobodan Jaric for inspiring our past, present and future research work. The study was supported in part by the Serbian Research Council under grant 175037.

\section{References}

Abernethy P, Wilson G, Logan P. Strength and power assessment. Issues, controversies and challenges. Sports Med, 1995; 19: 401-17

Allison SJ, Brooke-Wavell K, Folland JP. Multiple joint muscle function with ageing: the force-velocity and power-velocity relationships in young and older men. Aging Clin Exp Res, 2013; 25: 159-66

Bohannon RW. 2008. Hand-grip dynamometry predicts future outcomes in aging adults. J Geriatr Phys Ther 31: $3-10$

Bozic PR, Celik O, Uygur M, Knight CA, Jaric S. Evaluation of novel tests of neuromuscular function based on brief muscle actions. J Strength Cond Res, 2013; 27: 1568-78

Carriker CR. Components of Fatigue: Mind and Body. J Strength Cond Res, 2017; 31: 3170-76

Cronin JB, Mcnair PJ, Marshall RN. Force-velocity analysis of strength-training techniques and load: implications for training strategy and research. J Strength Cond Res, 2003; 17: 148-55

Cuk I, Markovic M, Nedeljkovic A, Ugarkovic D, Kukolj M, Jaric S. Force-velocity relationship of leg extensors obtained from loaded and unloaded vertical jumps. Eur J Appl Physiol, 2014; 114: 1703-14

Dempster WT, United States. Wright Air Development Division. Space requirements of the seated operator: geometrical, kinematic, and mechanical aspects of the body with special reference to the limbs. University of Michigan, Aero Medical Laboratory Contract No. AF 18(600)-43, Project No. 7214; 1956

Djuric S, Cuk I, Sreckovic S, Mirkov D, Nedeljkovic A, Jaric S. Selective effects of training against weight and inertia on muscle mechanical properties. Int J Sports Physiol Perform, 2016; 11: 927-32

Dobrijevic S, Ilic V, Djuric S, Jaric S. Force-velocity relationship of leg muscles assessed with motorized treadmill tests: Two-velocity method. Gait Posture, 2017; 56: 60-64

Driss T, Vandewalle H, Chevalier J-ML, Monod H. Force-velocity relationship on a cycle ergometer and knee-extensor strength indices. Can J Appl Physiol, 2002; 27: 250-62

Feeney D, Stanhope SJ, Kaminski TW, Machi A, Jaric S. Loaded vertical jumping: force-velocity relationship, work, and power. J Appl Biomech, 2016; 32: 120-27

García-Ramos A, Feriche B, Pérez-Castilla A, Padial P, Jaric S. Assessment of leg muscles mechanical 
capacities: Which jump, loading, and variable type provide the most reliable outcomes? Eur J Sport Sci, 2017; 17: 690-98

García-Ramos A, Jaric S, Padial P, Feriche B. Force-velocity relationship of upper body muscles: traditional versus ballistic bench press. J Appl Biomech, 2016; 32: 178-85

Golas A, Maszczyk A, Pietraszewski P, Stastny P, Tufano JJ, Zajac A. Effects of pre-exhaustion on the patterns of muscular activity in the flat bench press. J Strength Cond Res, 2017; 31(7): 1919-1924

Hahn D, Herzog W, Schwirtz A. Interdependence of torque, joint angle, angular velocity and muscle action during human multi-joint leg extension. Eur J Appl Physiol, 2014; 114: 1691-702

Jaric S. Force-velocity relationship of muscles performing multi-joint maximum performance tasks. Int $J$ Sports Med, 2015; 36: 699-704

Jaskolska A, Goossens P, Veenstra B, Jaskolski A, Skinner J. Comparison of treadmill and cycle ergometer measurements of force-velocity relationships and power output. Int J Sports Med, 1999; 20: 192-97

Jiménez-Reyes P, Samozino P, Brughelli M, Morin J-B. Effectiveness of an individualized training based on force-velocity profiling during jumping. Front Physiol, 2017; 7: 1-13

Jiménez-Reyes P, Samozino P, Cuadrado-Peñafiel V, Conceição F, González-Badillo JJ, Morin J-B. Effect of countermovement on power-force-velocity profile. Eur J Appl Physiol, 2014; 114: 2281-88

Limonta E, Sacchi M. Morphological analysis of force/velocity relationship in dynamic exercise at varying loads. J Strength Cond Res, 2010; 24: 2065-72

Mandic R, Jakovljevic S, Jaric S. Effects of countermovement depth on kinematic and kinetic patterns of maximum vertical jumps. J Electromyogr Kinesiol, 2015; 25: 265-72

Markovic S, Mirkov DM, Knezevic OM, Jaric S. Jump training with different loads: effects on jumping performance and power output. Eur J Appl Physiol, 2013; 113: 2511-21

Markovic S, Mirkov DM, Nedeljkovic A, Jaric S. Body size and countermovement depth confound relationship between muscle power output and jumping performance. Hum Mov Sci, 2014; 33: 203-10

McMaster DT, Gill N, Cronin J, McGuigan M. A brief review of strength and ballistic assessment methodologies in sport. Sports Med, 2014; 44: 603-23

Morin J, Samozino P, Bonnefoy R, Edouard P, Belli A. Direct measurement of power during one single sprint on treadmill. J Biomech, 2010; 43: 1970-75

Morin JB, Samozino P. Biomechanics of training and testing: Innovative concepts and simple field methods. Biomechanics of Training and Testing: Innovative Concepts and Simple Field Methods, 2018; Springer.

Newton RU, Murphy AJ, Humphries BJ, Wilson GJ, Kraemer WJ, Häkkinen K. Influence of load and stretch shortening cycle on the kinematics, kinetics and muscle activation that occurs during explosive upper-body movements. Eur J Appl Physiol Occup Physiol, 1997; 75: 333-42

Nikolaidis P. Age-and sex-related differences in force-velocity characteristics of upper and lower limbs of competitive adolescent swimmers. J Hum Kinet, 2012; 32: 87-95

Pazin N, Berjan B, Nedeljkovic A, Markovic G, Jaric S. Power output in vertical jumps: does optimum loading depend on activity profiles? Eur J Appl Physiol, 2013; 113: 577-89

Perez-Castilla A, Feriche B, Jaric S, Padial P, Garcia-Ramos A. Validity of a Linear Velocity Transducer for Testing Maximum Vertical Jumps. J Appl Biomech, 2017; 33: 388-92

Perez-Castilla A, Jaric S, Feriche B, Padial P, Garcia-Ramos A. Evaluation of Muscle Mechanical Capacities through the Two-load Method: Optimization of the Load Selection. J Strength Cond Res, 2018; 32(5): $1245-53$

Pojednic RM, Clark DJ, Patten C, Reid K, Phillips EM, Fielding RA. The specific contributions of force and velocity to muscle power in older adults. Exp Gerontol, 2012; 47: 608-13

Prebeg G, Cuk I, Suzovic D, Stojiljkovic S, Mitic D, Jaric S. Relationships among the muscle strength properties as assessed through various tests and variables. J Electromyogr Kinesiol, 2013; 23: 455-61 
Rabita G, Dorel S, Slawinski J, Sàez-de-Villarreal E, Couturier A, Samozino P, Morin JB. Sprint mechanics in world-class athletes: a new insight into the limits of human locomotion. Scand J Med Sci Sports, 2015; 25: 583-94

Rahmani A, Viale F, Dalleau G, Lacour J-R. Force/velocity and power/velocity relationships in squat exercise. Eur J Appl Physiol, 2001; 84: 227-32

Ravier G, Grappe F, Rouillon J. Application of force-velocity cycle ergometer test and vertical jump tests in the functional assessment of karate competitor. J Sports Med Phys Fitness, 2004; 44: 349

Sánchez-Medina L, González-Badillo JJ, Pérez CE, Pallarés JG. Velocity- and power-load relationships of the bench pull vs. Bench press exercises. Int J Sports Med, 2014; 35(3):209-16.

Samozino P, Edouard P, Sangnier S, Brughelli M, Gimenez P, Morin J-B. Force-velocity profile: imbalance determination and effect on lower limb ballistic performance. Int J Sports Med, 2014; 35: 505-10

Sreckovic S, Cuk I, Djuric S, Nedeljkovic A, Mirkov D, Jaric S. Evaluation of force-velocity and powervelocity relationship of arm muscles. Eur J Appl Physiol, 2015; 115: 1779-87

Van Den Tillaar R, Ettema G. A force-velocity relationship and coordination patterns in overarm throwing. J Sports Sci Med, 2004; 3: 211

Vandewalle H, Peres G, Heller J, Panel J, Monod H. Force-velocity relationship and maximal power on a cycle ergometer. Eur J Appl Physiol Occup Physiol, 1987; 56: 650-56

Wilson GJ, Murphy AJ. The use of isometric tests of muscular function in athletic assessment. Sports Med, 1996; 22: 19-37

Wilson GJ, Murphy AJ, Walshe AD, Ness K. Stretch shorten cycle performance: detrimental effects of not equating the natural and movement frequencies. Res Q Exerc Sport, 1996; 67: 373-79

Yamauchi J, Ishii N. Relations between force-velocity characteristics of the knee-hip extension movement and vertical jump performance. J Strength Cond Res, 2007; 21: 703

Zivkovic MZ, Djuric S, Cuk I, Suzovic D, Jaric S. Muscle force-velocity relationships observed in four different functional tests. J Hum Kinet, 2017a; 56: 39-49

Zivkovic MZ, Djuric S, Cuk I, Suzovic D, Jaric S. A simple method for assessment of muscle force, velocity, and power producing capacities from functional movement tasks. J Sports Sci, 2017b; 35: 1287-93

\section{Corresponding author:}

\section{Aleksandar Nedeljkovic}

University of Belgrade, Faculty of Sport and Physical Education,

Blagoja Parovica 156, 11030 Belgrade, Serbia.

Tel.: +381-64-2386001.

E-mail: aleksandar.nedeljkovic@fsfv.bg.ac.rs 